\title{
Genetic Studies in Primary Gout
}

\author{
INVESTIGATIONS ON THE PLASMA LEVELS \\ OF THE URATE-BINDING $\alpha_{1}-\alpha_{2}$-GLOBULIN IN \\ INDIVIDUALS FROM TWO GOUTY KINDREDS
}

\author{
J. O. Alvsaker \\ From the Department of Biochemistry, University of Oslo, Blindern, and the \\ Department of Medicine, Drammen Hospital, Drammen, Norway
}

A в S T R A C T The plasma levels of the uratebinding $\alpha_{1}-\alpha_{2}$-globulin, as determined by its uratebinding capacity, have been recorded in 19 individuals from two gouty kindreds. A significantly reduced binding capacity, accounting for 13-30\% of the mean value obtained in healthy, unrelated control subjects, was found in all cases of gout and in the single case of essential hyperuricemia included in the present study. In addition, six apparently healthy members of one of these kindreds also exhibited this characteristic. The distribution of the characteristic in three subsequent generations from this kindred further supported the hypothesis that the reduced binding capacity was inherited as an autosomal trait for which affected subjects were heterozygous.

Based on the present observation, the mechanisms of inheritance in primary gout are discussed with special emphasis on the possible cooperation of genetic and environmental factors.

\section{INTRODUCTION}

Although the familial nature of gout has been recognized since antiquity, its mode of transmission is still unknown as are the underlying specific metabolic defects. However, even since Talbott (1) reported a high incidence of elevated serum urate levels in asymptomatic relatives of patients with primary gout, this "essential" hyperuricemia has

Dr. Alvsaker is a fellow of the Norwegian Research Council for Science and the Humanities.

Received for publication 7 July 1967 and in revised form 6 December 1967. been regarded as an incomplete manifestation of the gouty trait. Consequently, most genetic studies in this field have concentrated upon the distribution of the blood urate levels in certain families and populations. The results initially obtained $(2,3)$ suggested that essential hyperuricemia was due to an autosomal trait, whereas more recent work (4) indicates multifactorial inheritance. In addition, the marked increase in the frequency of gout in the Maori people of New Zealand which accompanied their adoption to western civilization (5) is striking and suggests (6) the presence at least in this population of an inherited trait which predisposes them to hyperuricemia and gout and which is not manifest unless certain environmental conditions prevail. This interplay of genetic and environmental factors in primary gout has been further indicated by the observation of a significantly higher mean serum urate level in Filipinos living in the United States as compared to racially identical Filipinos staying on the Philippine islands (7). The precise inherited and environmental factors involved, however, remain to be demonstrated.

Although recent work $(8,9)$ has clarified the mechanisms by which gouty arthritis develops, the fact remains that only a relatively small fraction of hyperuricemic subjects develop gouty arthritis. Evidence for a possible modifying factor which could influence the expression of the disease by influencing the solubility of urate in plasma was provided by the author's demonstration of a uratebinding protein in human plasma (10). This protein, and $\alpha_{1}-\alpha_{2}-$ globulin, seemed to be absent from 
plasma of seven patients with primary gout (10), a finding which suggests that the protein deficiency might be another expression for the gouty trait. In the present study the concentration of this protein in plasma, as judged from its urate-binding capacity, has therefore been determined in individuals from two gouty kindreds.

\section{METHODS}

\section{Clinical material}

Plasma from 17 individuals belonging to a gouty kindred (the F-kindred) and from two gouty females belonging to another kindred (the G-kindred) was investigated. Control plasma from five healthy, unrelated individuals (among whom were the wives of two gouty brothers of the F-kindred) who were not hyperuricemic was used for the same isolation procedures.

The F-kindred originates from a circumscribed rural area in southern Norway. Reliable clinical accounts were obtained in individuals from four generations, and living subjects from three of these generations were available for the present study (Fig. 1).

The index case (generation III, No. 7) is a $48 \mathrm{yr}$ old truck driver who has suffered frequent attacks of acute gouty arthritis for the last $10 \mathrm{yr}$. $\mathrm{He}$ also exhibits tophi (verified by the murexide test), and his serum urate levels have repeatedly been in the range 8-12 $\mathrm{mg} / 100 \mathrm{ml}$. Urine examinations have hitherto failed to demonstrate proteinuria or formed elements by microscopy, and a urine specific gravity as high as 1.030 has been recorded. Serum creatinine levels have been between 0.9-1.2 $\mathrm{mg} / 100 \mathrm{ml}$. On two different occasions his renal uric acid excretion on a low purine diet has been 400 $450 \mathrm{mg} / 24 \mathrm{hr}$. The index case thus probably produces normal quantities of uric acid.

Two more cases of gout are known in this kindred. A brother of the index case (generation III, No. 5) exhibited an almost identical clinical picture of gout for which he has been hospitalized several times. In addition, the grandfather of the index case (generation I) suffered from classical podagra after he reached an age of $40 \mathrm{yr}$.

The daughter of the index case (generation IV, No. 6) has during the last $5 \mathrm{yr}$ suffered two attacks of acute arthritis localized to the left first metatarsophalangeal joint and lasting for about 10 days. On these occasions, however, no serum uric acid determinations were obtained nor did she receive any specific treatment with colchicine.

The subjects investigated were similar with respect to economic status, dietary habits, and level of activity except for the index case, his gouty brother, and another brother (generation III, No. 2) who were habitual beer drinkers. The only individual in this sibship not investigated (generation III, No. 4) died from acute meningitis in 1949.

The G-kindred originates from a coastal district in the northwestern part of Norway and has no relation to the F-kindred or its place of origin. The index case (Fig. 2) is a $21 \mathrm{yr}$ old nurse who has experienced severe, repeated attacks of migraine since she was $12 \mathrm{yr}$ old. At an age of 16, she got her first attack of acute arthritis localized to the first metatarsophalangeal joint of her left big toe. Since then she has suffered a few similar attacks for which she has been hospitalized. On these occasions her serum urate levels have repeatedly been in the range of 7-7.5 mg/100 ml. The response to colchicine was excellent. No protein or formed elements have hitherto been

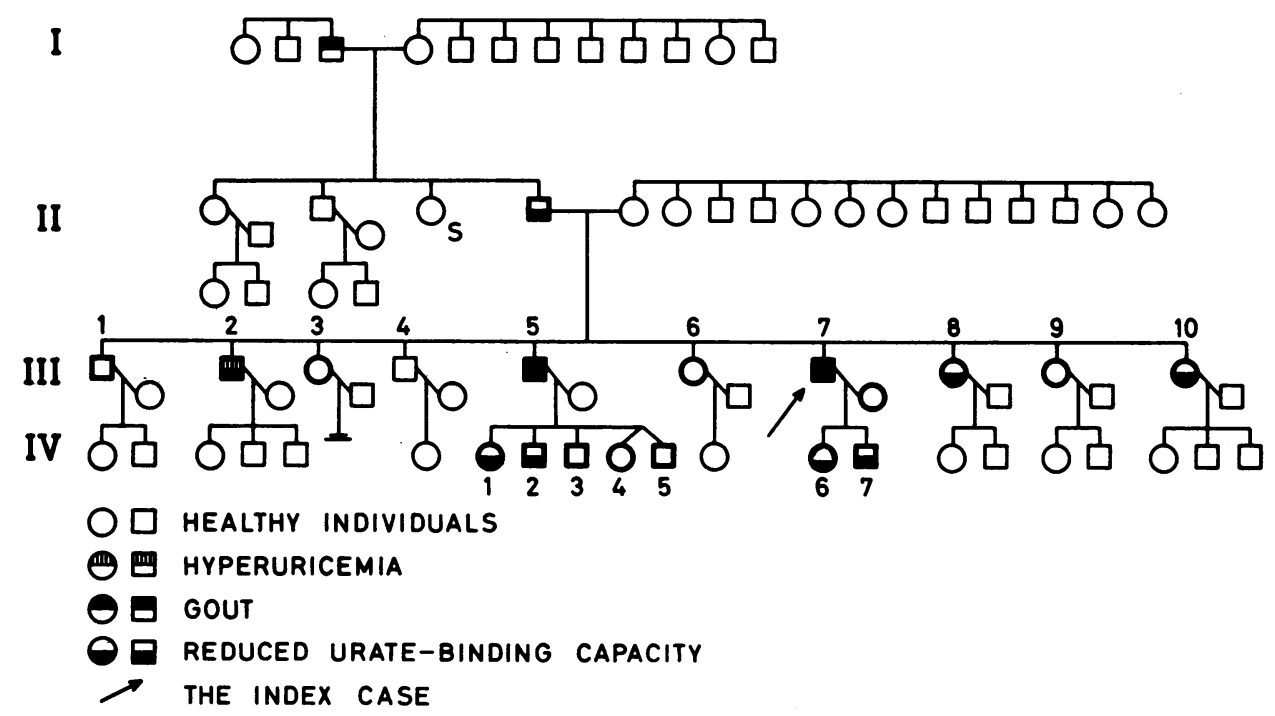

FIgURE 1 Pedigree of the F-kindred. Symbols drawn with thick lines represent individuals investigated, whereas symbols drawn with fine lines represent individuals not investigated but in whom reliable clinical accounts were obtained. 


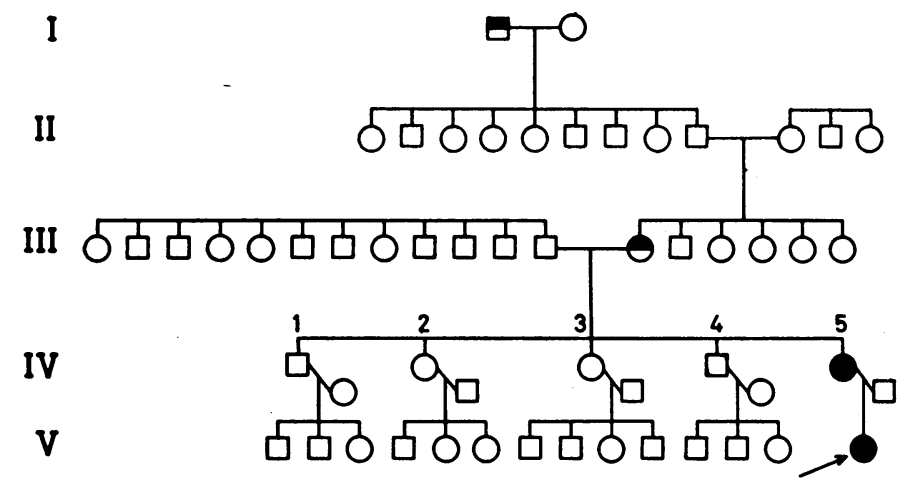

FIGURE 2 Pedigree of the G-kindred. The symbols are identical with those used for the F-kindred. demonstrated in her urine, and urine specific gravity as high as 1.028 has been recorded repeatedly. Her serum creatinine levels have been $0.7-1.1 \mathrm{mg} / 100 \mathrm{ml}$. Her renal uric acid excretion on a low purine diet, recorded on two different occasions, has been $620-700 \mathrm{mg} / 24 \mathrm{hr}$. The index case thus probably produces excessive quantities of uric acid.

Three more cases of gout are known in this kindred (Fig. 2). The mother of the index case (generation IV, No. 5) is a $45 \mathrm{yr}$ old housewife who has suffered frequent attacks of migraine since she was $13 \mathrm{yr}$ old. At an age of 16 , she had her first attack of acute arthritis localized to her left big toe. Her serum urate concentration at that time was $7.8 \mathrm{mg} / 100 \mathrm{ml}$. In the following years she was admitted to our university clinics several times because of attacks of acute arthritis with varying localization and with serum urate levels in the range of $8-14 \mathrm{mg} / 100$ $\mathrm{ml}$. She now shows severe, chronic tophaceous gout (murexide test) with nephropathy and hypertension. Except for migraine and a few attacks of epilepsy (grand mal) no other disease underlying her gout has ever been demonstrated. At the time when the plasma was collected, this patient was on allopurinol treatment with excellent response.

The grandmother of the index case (generation III) who died from acute cerebral hemorrhage at age 72 had migraine since she was $12 \mathrm{yr}$ old and had repeated attacks of acute arthritis localized to both her big toes since after age 50. In addition, one male in this kindred (generation I) had classical podagra.

A striking aggregation of neurological disorders seems to occur in the G-kindred. Thus, in generation IV, No. 2 is suffering from narcolepsy, No. 3 from epilepsy and migraine, and No. 4 from migraine, whereas in generation III one case of migraine and one case of epilepsy are known.

No consanguinous matings or any evidence of inbreeding could be found in the two gouty kindreds studied.

Plasma collection. Whole blood was withdrawn into sterile tubes containing heparin to a final concentration of $10 \mathrm{U} / \mathrm{ml}$ and centrifuged immediately at $1000 \mathrm{~g}$ for $15 \mathrm{~min}$ at $0^{\circ} \mathrm{C}$. The plasma was drawn off and stored at $-20^{\circ} \mathrm{C}$ until examined (not more than $8 \mathrm{wk}$ ). Analysis on fresh and stored plasma showed that storage did not influence the results.

Uric acid determinations were all carried out by enzymatic differential spectrophotometry (11) with a Beckman DB spectrophotometer (light path, $10 \mathrm{~mm}$ ). Uricase from Leo Pharmaceutical Products Trading Ltd., Copenhagen, Denmark was employed.

Assay of the maximum urate-binding capacity of plasma. The modification of the equilibrium gel filtration method of Hummel and Dreyer (12) used in these studies has been described in detail in previous reports $(13,14)$. In order to record the maximum urate-binding capacity the urate concentration in the eluant was kept at $10 \mathrm{mg} /$ $100 \mathrm{ml}$.

Immunoelectrophoresis ${ }^{1}$ was carried out according to the micromethod of Scheidegger (15) in sodium barbital buffer, $\mathrm{pH} 8.6(\mu=0.05)$. The protein staining was performed in a saturated solution of amido-black $10 \mathrm{~B}$ (E. Merck, Darmstadt, Germany) in methanol : distilled water:acetic acid $(50: 50: 10)$ followed by destaining in distilled water.

Antiserum. Rabbit immune serum prepared against the urate-binding protein fraction as previously described (10) was used.

Radioautography. The protein fractions to be investigated were dissolved in a $0.9 \%(\mathrm{w} / \mathrm{v})$ solution of aqueous sodium chloride containing $40 \mu \mathrm{c} / \mathrm{ml}$ of uric acid- $2-{ }^{14} \mathrm{C}$ (Radiochemical Centre, Amersham, England). After the precipitation arcs had developed, the agar plates were dried in the air at $37^{\circ} \mathrm{C}$ and placed in firm contact with Kodak x-ray film (Kodak occlusal Ultraspeed, Code Df. 45) for 4-6 wk. In order to exclude the possibility that chemical reactions which eventually take place between proteins and the film material might give "false" positive results, identical protein solutions, except for the presence of uric acid- $2-{ }^{14} \mathrm{C}$, were used as blanks.

The partial isolation of the urate-binding $\alpha_{1}-\alpha_{2}-$ globulin. $O$-diethylaminoethyl (DEAE)-Sephadex (A-50, medium grade, AB Pharmacia, Stockholm, Sweden) prepared for use as described by the manufacturers was packed in columns $(3 \mathrm{~cm} \times 15 \mathrm{~cm})$ and equilibrated with $0.01 \mathrm{M}$ sodium phosphate buffer, $\mathrm{pH}$ 7.35. The plasma proteins

1 The equipment of LKB-Produkter AB, Stockholm, Sweden, was employed. 
TABLE 1

Survey of Plasma Urate Levels and Maximum Urate-Binding Capacties in Individuals Studied

\begin{tabular}{|c|c|c|c|c|c|c|}
\hline Individuals & Sex & Age & $\begin{array}{c}\text { Plasma } \\
\text { uric acid }\end{array}$ & $\begin{array}{c}\text { Urate } \\
\text { bound } \\
\text { by } 100 \mathrm{ml} \\
\text { of plasma }\end{array}$ & $\begin{array}{l}\text { Weight of } \\
\text { the urate- } \\
\text { binding } \\
\text { protein } \\
\text { fraction }\end{array}$ & $\begin{array}{l}\text { Total amount } \\
\text { of urate } \\
\text { bound by } \\
\text { the isolated } \\
\text { urate-binding } \\
\text { protein frac- } \\
\text { tion from } \\
100 \mathrm{ml} \text { of } \\
\text { plasma }\end{array}$ \\
\hline & & $y r$ & $m g / 100 \mathrm{ml}$ & $m g$ & $\underset{\text { plasma }}{m g / 100 m l}$ & $\mu g$ \\
\hline \multicolumn{7}{|l|}{ Healthy subjects } \\
\hline *E.F. & F & 57 & 4.25 & 4.0 & 100 & 400 \\
\hline *S.F. & F & 45 & 2.50 & 3.7 & 90 & 360 \\
\hline J.M. & $\mathbf{M}$ & 53 & 4.25 & 3.8 & 92 & 405 \\
\hline P.N. & F & 47 & 4.00 & 3.7 & 86 & 353 \\
\hline R.S. & $\mathbf{M}$ & 54 & 3.50 & 3.6 & 98 & 412 \\
\hline \multicolumn{7}{|l|}{ The F-kindred } \\
\hline A.F., generation II, & $\mathbf{M}$ & 83 & 5.50 & 2.8 & 70 & 63 \\
\hline B.F., generation III, No. 1 & $\mathbf{M}$ & 58 & 4.50 & 4.2 & 102 & - 428 \\
\hline J.F., generation III, No. 2 & $\mathbf{M}$ & 57 & 7.75 & 2.6 & 68 & 54 \\
\hline A.K., generation III, No. 3 & $\mathrm{~F}$ & 56 & 4.25 & 3.7 & 90 & 405 \\
\hline †A.F., generation III, No. 5 & $\mathbf{M}$ & 50 & 9.25 & 2.8 & 69 & 55 \\
\hline R.V., generation III, No. 6 & $\mathrm{~F}$ & 49 & 5.00 & 3.9 & 70 & 364 \\
\hline$\ddagger$ R.F., generation III, No. 7 & $\mathbf{M}$ & 48 & 8.50 & 2.6 & 79 & 85 \\
\hline G.V., generation III, No. 8 & F & 45 & 4.00 & 3.0 & 72 & 100 \\
\hline S.R., generation III, No. 9 & $\mathbf{F}$ & 42 & 3.50 & 4.0 & 78 & 436 \\
\hline K.D., generation III, No. 10 & $\mathrm{~F}$ & 40 & 3.75 & 2.6 & 65 & 78 \\
\hline K.J., generation IV, No. 1 & $\mathbf{F}$ & 26 & 5.25 & 2.9 & 66 & 52 \\
\hline O.F., generation IV, No. 2 & $\mathbf{M}$ & 20 & 5.75 & 2.9 & 82 & 120 \\
\hline V.F., generation IV, No. 3 & $\mathbf{M}$ & 18 & 6.00 & 4.3 & 105 & 470 \\
\hline K.F., generation IV, No. 4 & $\mathbf{F}$ & 16 & 4.25 & 3.8 & 90 & 405 \\
\hline H.F., generation IV, No. 5 & $\mathbf{M}$ & 16 & 4.50 & 4.1 & 78 & 367 \\
\hline S.H., generation IV, No. 6 & $\mathbf{F}$ & 23 & 4.75 & 2.6 & 100 & 75 \\
\hline R.F., generation IV, No. 7 & $\mathbf{M}$ & 19 & 6.50 & 2.9 & 76 & 94 \\
\hline \multicolumn{7}{|l|}{ The G-kindred } \\
\hline †J.D., generation IV & $\mathbf{F}$ & 45 & 4.008 & 2.4 & 82 & 85 \\
\hline$\ddagger \mathrm{K} . \mathrm{O}$., generation V & F & 21 & 7.00 & 2.6 & 93 & 52 \\
\hline
\end{tabular}

* Wives of Nos. 5 and 7, the F-kindred.

$\ddagger$ Gouty subjects.

\& Allopurinol treated.

(from $60-\mathrm{ml}$ plasma samples) were equilibrated with this buffer by gel filtration on suitable Sephadex G-50 coarse grade (AB Pharmacia, Stockholm, Sweden) columns and subsequently transferred to the DEAE-Sephadex columns. Elution was now carried out with the sodium phosphate buffer containing $0.20 \mathrm{M}$ sodium chloride. When the absorbance of the effluent $(280 \mathrm{~m} \mu)$ approached 0 , elution was continued with buffer containing $0.40 \mathrm{M}$ sodium chloride. The columns were finally eluted with buffer containing $1 \mathrm{M}$ sodium chloride (10). These procedures were carried out at $+4^{\circ} \mathrm{C}$.

The protein fractions corresponding to the different steps of elution respectively were partly desalted by dialysis against distilled water for $48 \mathrm{hr}$, lyophilized, and finally completely desalted by gel filtration on suitable G-50 coarse grade Sephadex columns with distilled water as the eluant. After relyophilization the weight of the individual protein fractions was recorded. In the remainder of this paper, for the purpose of convenience, the protein fraction eluted from the DEAE-Sephadex columns with buffer containing $0.40 \mathrm{M}$ sodium chloride will be termed "the urate-binding protein fraction."

The maximum urate-binding capacity of the urate-binding protein fraction. The procedures and columns employed were identical with those used for recording the maximum plasma urate-binding capacities. Completely desalted, lyophilized protein material $(5 \mathrm{mg}$ ) was dissolved in $200 \mu 1$ of the urate-containing sodium phosphate buffer (the eluant) and investigated.

\section{RESULTS}

Plasma urate levels. In the United States and most of Europe the upper limits of normal blood uric acid levels (the enzymatic spectrophotometric method) are, statistically defined, $6.9-7.5 \mathrm{mg} / 100$ $\mathrm{ml}$ in males and $5.7-6.6 \mathrm{mg} / 100 \mathrm{ml}$ in females (16). According to this definition all gouty subjects studied (Table I) except one (G-kindred, 
generation IV, No. 5), who was taking allopurinol, demonstrated hyperuricemia at the time when plasma was collected. As shown in this table only one individual in the present study (F-kindred, generation III, No. 2) exhibited essential hyperuricemia. On the other hand, the young female (F-kindred, generation IV, No. 6) with two attacks of acute arthritis demonstrated serum urate levels within the normal range (determined twice with a 6 month interval). Although there are reports of a few individuals who have abortive attacks of gouty arthritis and are restored for long periods to normal blood urate levels (17), this case can hardly be recorded as gout without further observation. In two more individuals belonging to the F-kindred (the father of the index case and generation IV, No. 7) the plasma urate levels were determined twice (with a 6 month interval). In these cases the values obtained were markedly constant with a maximum variation of not more than $\pm 0.25 \mathrm{mg} / 100 \mathrm{ml}$, although no dietary restrictions were undertaken.

The maximum plasma urate-binding capacities. In a previous study (14) it was found that the maximum urate-binding capacity of plasma in 10 healthy subjects (at a "free" urate concentration of $10 \mathrm{mg} / 100 \mathrm{ml}$ ) varied from 3.4 to $4.4 \mathrm{mg} / 100$ $\mathrm{ml}$, with an arithmetic mean value of $3.9 \mathrm{mg}$. In no case did analysis in duplicate vary by more that $0.5 \mathrm{mg} / 100 \mathrm{ml}$ of plasma. The normal range for the binding capacity in healthy individuals was further defined as $3.1-4.7 \mathrm{mg}$ (arithmetic mean value $\pm 3 \mathrm{SD})$. The results obtained in the present study (mean value of analysis in duplicate) are shown in Table I. From this table it will be seen that the maximum urate-binding capacities of plasma from five additional healthy subjects studied all fall within the normal range. Significantly reduced binding capacities, on the other hand, were found in all gouty subjects investigated, in the single individual exhibiting essential hyperuricemia, and in six apparently healthy individuals belonging to the F-kindred. In addition, the young female (F-kindred, generation IV, No. 6) with two attacks of acute arthritis demonstrated a significantly reduced urate-binding capacity.

Partial isolation of the urate-binding $\alpha_{1}-\alpha_{2}-$ globulin. In the present work the procedure for partial isolation of the urate-binding $\alpha_{1}-\alpha_{2}$-globulin previously described (10) was modified so that gel filtration of plasma on Sephadex G-200 columns was avoided. This step was omitted because the low protein recovery (about 12\%) previously obtained made the estimation of quantitative variations (of major interest in genetic studies) difficult. In order to insure that this modification did not influence the elution of the urate-binding $\alpha_{1}-\alpha_{2}$-globulin from DEAE-Sephadex, the following experiments were carried out : lyophilized protein material corresponding to the three different steps of elution (DEAE-Sephadex) respectively was dissolved in a $0.9 \%(\mathrm{w} / \mathrm{v})$ aqueous solution of sodium chloride to a final concentration of $5 \%$ $(\mathrm{w} / \mathrm{v})$ and examined by immunoelectrophoresis. A distinct precipitation arc representing the uratebinding $\alpha_{1}-\alpha_{2}$-globulin could be detected only in material from the urate-binding protein fraction. Thus, in accordance with previous observations (10) this protein seems almost exclusively to be eluted in this fraction.

The weights of the urate-binding protein fractions from the different subjects studied are shown in Table I. Although there seems to be a tendency for subjects demonstrating a reduced plasma uratebinding capacity to have low total amounts of protein there are more exceptions to this tendency which prevent any further conclusions. It will be seen (Table I) that the total amount of protein in individuals with a plasma urate-binding capacity within normal limits is from 70 to $105 \mathrm{mg} / 100 \mathrm{ml}$ of plasma, with an arithmetic mean value of 89.9 $\mathrm{mg}$ as compared to $42.5 \mathrm{mg}$ in the previous study (10). The total amount of urate bound by the urate-binding protein fractions are also shown in Table I. The maximum variation from the mean value of analysis in duplicate was $\pm 45 \mu \mathrm{g}$. It will be seen that this amount in individuals with a plasma urate-binding capacity within normal limits varies between 352 and $470 \mu \mathrm{g}$, with a mean value of $400 \mu \mathrm{g}$, as compared to $127.5 \mu \mathrm{g}$ in the previous study (10). It therefore may be calculated that the present modification of the isolation procedure (urate-binding protein fraction) has resulted in an increase in the recovery of the uratebinding $\alpha_{1}-\alpha_{2}$-globulin from about $12 \%$ (10) to $37-38 \%$.

On the contrary, individuals with significantly reduced plasma urate-binding capacities showed values for bound urate (Table I) between 52 and $120 \mu \mathrm{g}$, thus corresponding to $13-30 \%$ of the mean 


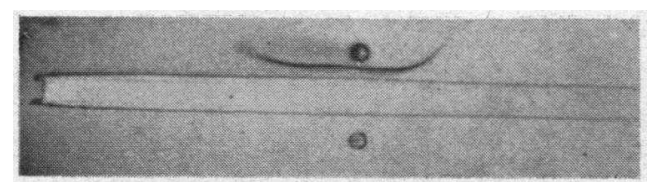

FIGURE 3 Photograph of a radioautogram (immunoelectrophoresis, anode to the left) demonstrating the binding of the uric acid-2- ${ }^{14} \mathrm{C}$ to the urate-binding $\alpha_{1}-\alpha_{2}-$ globulin.

value found in subjects with plasma urate-binding capacities within normal limits. However, since the maximum variation from the mean value of analysis in duplicate could be as much as $\pm 45 \mu \mathrm{g}$, it is evident that the present method does not permit any further subdivision within the group with low binding capacities. On the other hand, it clearly distinguishes between affected and nonaffected individuals.

Immunoelectrophoretical studies on the uratebinding protein fractions revealed the presence of the urate-binding $\alpha_{1}-\alpha_{2}$-globulin in plasma from all subjects studied. Parallel experiments carried out in the presence of uric acid- $2-{ }^{14} \mathrm{C}$ also demonstrated a radioactive precipitation arc corresponding to this protein in all cases (Fig. 3). Thus, in obvious disagreement with previous findings (10), the urate-binding $\alpha_{1}-\alpha_{2}$-globulin is present in plasma from individuals with primary gout among whom are two subjects (F-kindred, generation III, Nos. 5 and 7) included in the previous work (10). In order to rule out the possibility that this controversial finding might be due to spontaneous variations in the plasma levels of this protein in gouty individuals, the following experiment was carried out: plasma from the two gouty subjects mentioned above, that had been stored at $-20^{\circ} \mathrm{C}$ since the original study (10), was reinvestigated by the modified isolation procedure. In both cases the urate-binding $\alpha_{1}-\alpha_{2}$-globulin now could be detected. This observation strongly indicates that the controversial finding, mentioned above, is due to the difference in protein recovery obtained by the two different isolation procedures.

\section{DISCUSSION}

In accordance with previous findings $(10,14)$, all gouty individuals included in the present study demonstrated a significantly reduced plasma uratebinding capacity. This obviously is due to a decreased binding capacity of a plasma protein sub- fraction cuntaining the urate-binding $\alpha_{1}-\alpha_{2}$-globutlin. Whether the reduced binding capacity results from a faulty synthesis of this protein, the production of an altered protein with lower urate-binding capacity, or a combination of these possibilities remain to be seen. Consequently, for the following genetic considerations, a binding capacity of the protein fraction containing the urate-binding $\alpha_{1}-\alpha_{2}-$ globulin, in the range $13-30 \%$ of the values found in control subjects, has been used as the characteristic.

As will be seen from Fig. 1 the distribution of this characteristic has been recorded in two complete family groups belonging to the F-kindred (the families of generation III, Nos. 5 and 7). In both families the father demonstrates a reduced binding capacity and transfers it to children of both sexes, whereas the mother and some of the children have binding capacities within normal limits. This observation is consistent with the interpretation that the characteristic results from an autosomal trait for which affected individuals are heterozygous. Based on this assumption the ratio of affected-to-nonaffected subjects in the members of generation III should be 1 (ideal conditions). The ratio actually obtained is 1.25 . One member of this sibship (No. 4) was not studied. If he was nonaffected, the ideal ratio would be obtained. If he, on the contrary, was affected the ratio would be 1.5. However, the probability for obtaining such a ratio by chance in a sibship of 10 is rather high $(P=0.50[18])$. Since all gouty subjects studied so far demonstrate the actual characteristic it seems highly probable that it has been transferred to generation III from their gouty grandfather (generation I) through their father (generation II). In accordance with this assumption the father (generation II) also exhibits the characteristic. Thus, the present observations all support the hypothesis that the reduced urate-binding capacity is inherited in the F-kindred as an autosomal trait for which individuals affected are heterozygous.

The possibility of genetic considerations in the G-kindred, on the other hand, is rather limited because only two individuals belonging to this kindred have been available for studies on plasma proteins. These two subjects have been included in the present work primarily to see whether the defective urate-binding capacity might be limited 
to a smaller group of patients with primary gout. In this respect subjects from the G-kindred seem to represent a type of primary gout markediy different from that seen in the F-kindred, because the affected individuals are mainly young females and the index case probably a uric acid overproducer. The values for the urate-binding capacities obtained in the two gouty females from the G-kindred, however, were in the same range as found in gouty subjects from the F-kindred. In a previous study (10), five unrelated individuals with primary gout also exhibited the deficiency of the urate-binding globulin. These observations therefore suggest that the defective urate-binding capacity is a widespread defect in primary gout.

The relationship between significantly reduced plasma urate-binding capacities and the blood urate levels, on the other hand, is more obscure. In the present study only one individual (F-kindred, generation III, No. 2) demonstrated essential hyperuricemia. This subject also exhibited a significantly reduced urate-binding capacity. Six more individuals from the F-kindred (the father of the index case, generation III, Nos. 8 and 10 and generation IV, Nos. 1, 6, and 7) demonstrated reduced binding capacities. None of these subjects, however, has hitherto demonstrated hyperuricemia. This observation therefore may indicate that multifactorial inheritance in the F-kindred is necessary for gout and hyperuricemia to become manifest (4). Another possibility (6) is that the defective urate-binding capacity in this kindred is an inherited defect which requires the cooperation of physiological and environmental factors before hyperuricemia and gout develop. Regarding physiological factors, essential hyperuricemia in females is frequently delayed until menopause (16). None of the asymptomatic females in the F-kindred who exhibits reduced uratebinding capacities has reached this phase of life. Further, if environmental factors similar to those proposed in the Maori people of New Zealand (5) and the Filipinos living in the United States (7) are operating in the F-kindred, it is interesting to note that both gouty males in generation III as well as their hyperuricemic brother are habitual beer drinkers (19).

The striking feature in the G-kindred is that only females have suffered gout in three subsequent generations, and that the disease in two of them developed shortly after puberty. Common to these females, however, is that they all suffered heavy, repeated attacks of migraine from an age of 12-13 yr resulting in an almost continuous intake of salicylate-containing analgesics. Since it seems well established that low plasma levels of salicylate result in a reduced renal excretion of uric acid (20), salicylate ingestion may be a factor of considerable importance in these gouty females in addition to overproduction of urate.

The present findings in individuals belonging to two gouty kindreds thus may be in accordance with the hypothesis for the mechanisms of inheritance in primary gout proposed by Blumberg (6). On the other hand, based on the striking accumulation of neurological disorders in the G-kindred, it may be postulated that the early occurrence of gout in females from this kindred is due to the cooperation of several mutant genes. For the clarification of these problems, however, further investigations on the possible physiological functions of the urate-binding $\alpha_{1}-\alpha_{2}$-globulin (21) are required.

\section{ACKNOWLEDGMENTS}

It is a pleasure for me to thank Professor S. Laland for providing excellent working facilities and $\mathrm{Dr}$. O. Römcke for his kind interest in this work. I also want to thank Dr. H. Palmer and his staff, Drammen Hospital, and Dr. H. Chr. Godal, Ullevaal Hospital, for supplying the plasma samples. The author is much obliged to Dr. J. E. Seegmiller and Dr. M. Greene, National Institute of Arthritis and Metabolic Diseases, Bethesda, Md., for their kind assistance in reviewing the manuscript.

This work has in part been supported by grants from Freia Research Foundation and the Norwegian Rheumatism Council.

\section{REFERENCES}

1. Talbott, J. H. 1940. Serum urate in relatives of gouty patients. J. Clin. Invest. 19: 645.

2. Smyth, C. J., C. W. Cotterman, and R. H. Freyberg. 1948. The genetics of gout and hyperuricemia-an analysis of nineteen families. J. Clin. Invest. 27: 749.

3. Stecher, R. M., A. H. Hersh, and W. M. Solomon. 1949. The heredity of gout and its relationship to familial hyperuricemia. Ann. Internal Med. 31: 595.

4. Hauge, M., and B. Harvald. 1955. Heredity in gout and hyperuricemia. Acta Med. Scand. 152: 247.

5. Prior, I. A. M., B. S. Rose, H. P. B. Harvey, and F. Davidson. 1966. Hyperuricæmia, gout, and diabetic abnormality in Polynesian people. Lancet. 1: 333.

6. Blumberg, B. S. 1965. Heredity of gout and hyperuricemia. Arthritis Rheumat. 8: 627. 
7. Healey, L. A., M. D. Skeith, J. L. Decker, and P. S. Bayani-Sioson. 1967. Hyperuricemia in Filipinos: interaction of heredity and environment. $\mathrm{Am}$. J. Human Genct. 19: 81.

8. Seegmiller, J. E., and R. R. Howell. 1962. The old and new concepts of acute gouty arthritis. Arthritis Rheumat. 5: 616.

9. Faires, J. S., and D. J. McCarty, Jr. 1962. Acute arthritis in man and dog after intrasynovial injection of sodium urate crystals. Lancet. 2: 682.

10. Alvsaker, J. O. 1966. Uric acid in human plasma. V. Isolation and identification of plasma proteins interacting with urate. Scand. J. Clin. Lab. Invest. 18: 227.

11. Praetorius, E., and H. Poulsen. 1953. Enzymatic determination of uric acid. Scand. J. Clin. Lab. Invest. 5: 273.

12. Hummel, J. P., and W. J. Dreyer. 1962. Measurement of protein-binding by gel filtration. Biochim. Biophys. Acta. 63: 530.

13. Alvsaker, J. O. 1965. Uric acid in human plasma. III. Investigations on the interaction between the urate ion and human albumin. Scand. J. Clin. Lab. Invest. 17: 467.

14. Alvsaker, J. O. 1965. Uric acid in human plasma. IV. Investigations on the interaction between urate and the macromolecular fraction in plasma from healthy individuals and patients with diseases associated with hyperuricemia. Scand. J. Clin. Lab. Invest. $17: 476$.

15. Scheidegger, J. J. 1955. Une micro-méthode de l'immuno-électrophorese. Intern. Arch. Allergy Appl. Immunol. 7: 103.

16. Wyngaarden, J. B. 1966. Gout. In The Metabolic Basis of Inherited Disease. J. B. Stanbury, J. B. Wyngaarden, and D. S. Fredrickson, editors. McGraw-Hill Book Company, New York. 2nd edition. 667.

17. Hoffman, W. S. 1959. Some unsolved problems of gout. Med. Clin. North Am. 43: 595.

18. Stern, C. 1960. Principles of Human Genetics. W. H. Freeman \& Co., Publishers, San Francisco. 2nd edition. 142.

19. Maclachlan, M. J., and G. P. Rodnan. 1967. Effect of food, fast and alcohol on serum uric acid and acute attacks of gout. Am. J. Med. 42: 38.

20. Yü, T. F., and A. B. Gutman. 1959. Study of the paradoxical effects of salicylate in low, intermediate and high dosage on the renal mechanisms for excretion of urate in man. J. Clin. Invest. 38: 1298.

21. Alvsaker, J. O. 1966. Uric acid in human plasma. A contribution to the study of pathogenetic mechanisms in gout. Doctoral thesis. Universitetsforlaget, Oslo. 